\title{
RESEARCH HIGHLIGHT Ubiquitilated Fanconi ID complex embraces DNA
}

\author{
Yathish Jagadheesh Achar (DD) ${ }^{1}$ and Marco Foiani ${ }^{1,2}$ \\ Cell Research (2020) 30:554-555; https://doi.org/10.1038/s41422-020-0345-2
}

\begin{abstract}
Mono-ubiquitination of the Fanconi ID complex is essential for inter-strand DNA repair and thereby prevents Fanconi anemia, a genetic disorder with high cancer risk. Using cryo-EM, Wang et al. and Alcon et al. compared monoubiquitinated and non-ubiquitinated ID compelexes and found that upon mono-ubiquitination, the ID complex undergoes conformational changes to form a ring encircling DNA.
\end{abstract}

Replication fork stability in response to inter-strand DNA crosslink (ICL) depends on the Fanconi anemia (FA) repair pathway, which triggers mono-ubiquitination of the (ID) complex formed by $\mathrm{FANCI}$ and FANCD2. The ID complex preferentially binds to branched DNA structures including replication forks, flapDNA and Holliday junctions. ${ }^{1}$ Mono-ubiquitination of the ID (ubID) complex is crucial for ICL repair, replication fork stability and homologous recombination. Failure in ID ubiquitination causes developmental abnormalities, bone marrow failure and cancer predisposition, hallmarks of the FA syndrome. Since the ub-ID complex represents the active form, it was widely speculated that ub-ID has higher affinity for other DNA repair proteins, although mechanistic details on the consequences of ID ubiquitination were elusive.

Two recent studies published in Nature ${ }^{2}$ and Nat. Struct. Mol. Biol. ${ }^{3}$ provide structural details of ub-ID. Both studies compared the structures of ubiquitilated and non-ubiquitilated ID complexes from human ${ }^{2}$ and chicken ${ }^{3}$ cells. They found that a single ubiquitin moiety on FANCD2 triggers rearrangement of the ID complex. A previous report showed that non-ubiquitinated ID complex forms an open channel through hetero-dimerization in the presence of DNA and that both subunits shared structural similarities. ${ }^{4}$ Interestingly, the ubiquitination site as well as the regulatory phosphorylation sites were mapped at the dimerization interface. Although ubiquitination and its subsequent removal are crucial steps for completion of $I C L$, it remained a mystery how the FA core complex could access such buried sites. Based on these new studies, we now know that $\mathrm{FANCl}$ alone is sufficient to bind DNA through its basic groves and helical domains located at the $\mathrm{N}$ - and $\mathrm{C}$-termini, respectively, whereas FANCD2 binds to DNA through its flexible C-terminal domain. Moreover, they found that mono-ubiquitination induces structural changes in the ID complex, converting an open channel into a circular closed ring embracing DNA. Critical to this transition are the FANCD2-FANCI N-terminal interface acting as a hinge and the ubiquitin moiety at the back of FANCD2 forming a pin joint. This hinge movement allows the C-terminal domains of FANCD2 and FANCI to swing towards each other to form a tight clamp on DNA. FANCD2 ubiquitination is essential for the FA pathway and it is necessary and sufficient for the formation of a stable clamp, thus suggesting that $\mathrm{FANCl}$ ubiquitination plays a minor role in $\mathrm{ICL}$ repair.
Alcon et al. also showed that FANCD2 can form a homodimer that cannot bind to DNA or undergo ubiquitination. $\mathrm{FANCl}$ competes with one FANCD2 monomer allowing the formation of a stable-open-ID complex that can bind to DNA (Fig. 1). This implicates the FANCD2 homodimer complex in an autoregulatory loop that restricts the formation of a pre-active ID complex, in the absence of DNA damage. Interestingly, phosphorylation of FANCD2 by casein kinase 2 inhibits ubiquitination and ID complex formation. ${ }^{5}$ Moreover, ATR-mediated phosphorylation of $\mathrm{FANCl}$ acts as major molecular switch to activate the FA pathway upon replication stress, ${ }^{6}$ thus suggesting that $\mathrm{FANCl}$ phosphorylation is a key event that counteracts FANCD2 homodimerization (Fig. 1).

Wang et al. found that the ub-ID complex once bound to DNA loses its capability to recognize branched DNA structures and slides on the DNA, contributing to the formation of filamentous nucleoprotein arrays that have been recently described. ${ }^{7}$ The ubID clamp is very stable and persists as a chromatin-tethered complex in the absence of the USP1-UAF1 de-ubiquitination enzyme complex. ${ }^{8}$ De-ubiquitination of FANCD2 releases the clamp from DNA, representing an essential step for completion of $I C L$ repair. Failures in removing the ub-ID clamp from DNA might cause genome instability through off-target repair process or even generate a protein-DNA obstacle hindering replication and transcription. Similar to ubiquitination, de-ubiquitination of the ID complex occurs only in the presence of DNA, as UAF1 binding to DNA is crucial. $^{9}$ Thus, these results suggest that both ubiquitination and de-ubiquitination of the ID complex are regulated at the DNA level. Moreover, as the ATR-dependent FANCl phosphorylation is crucial for ID complex formation and ubiquitination, $\mathrm{FANCl}$ dephosphorylation is also critical for deubiquitination of FANCD2. ${ }^{10}$

FA-mediated repair occurs mainly during replication, whereas ICLs outside of $S$ phase are repaired by the NER pathway. Once fork progression is halted by ICL, FA facilitates several events including $\mathrm{ICL}$ recognition, nucleolytic cleavage, translesion synthesis across the damage, ICL removal and recruitment of homologous recombination factors. FA also protects stalled replication forks from the action of nucleases by stabilizing RAD51 filament formation. ${ }^{11}$ While the FA pathway senses ICL by means of stalled replication forks, it is unclear how ICLs can be sensed in the absence of stalled fork. However, recent observations showing that the ID complex co-localizes with R-loop structures and the defect in FA pathway elevates R-loops levels ${ }^{12}$ suggest that stalled transcription may facilitate ICL sensing. The association of the ID complex with common fragile sites containing highly expressed and large genes also suggests a role for this complex in coordinating $S$ phase.

Overall, the new findings on the conformational changes of the ID complex following mono-ubiquitination have relevant

${ }^{1}$ IFOM (Fondazione Istituto FIRC di Oncologia Molecolare) Via Adamello 16, 20139 Milan, Italy and 2Università degli Studi di Milano, Milano, Italy

Correspondence: Yathish Jagadheesh Achar (yathish.achar@ifom.eu) or Marco Foiani (marco.foiani@ifom.eu)

Published online: 29 May 2020 


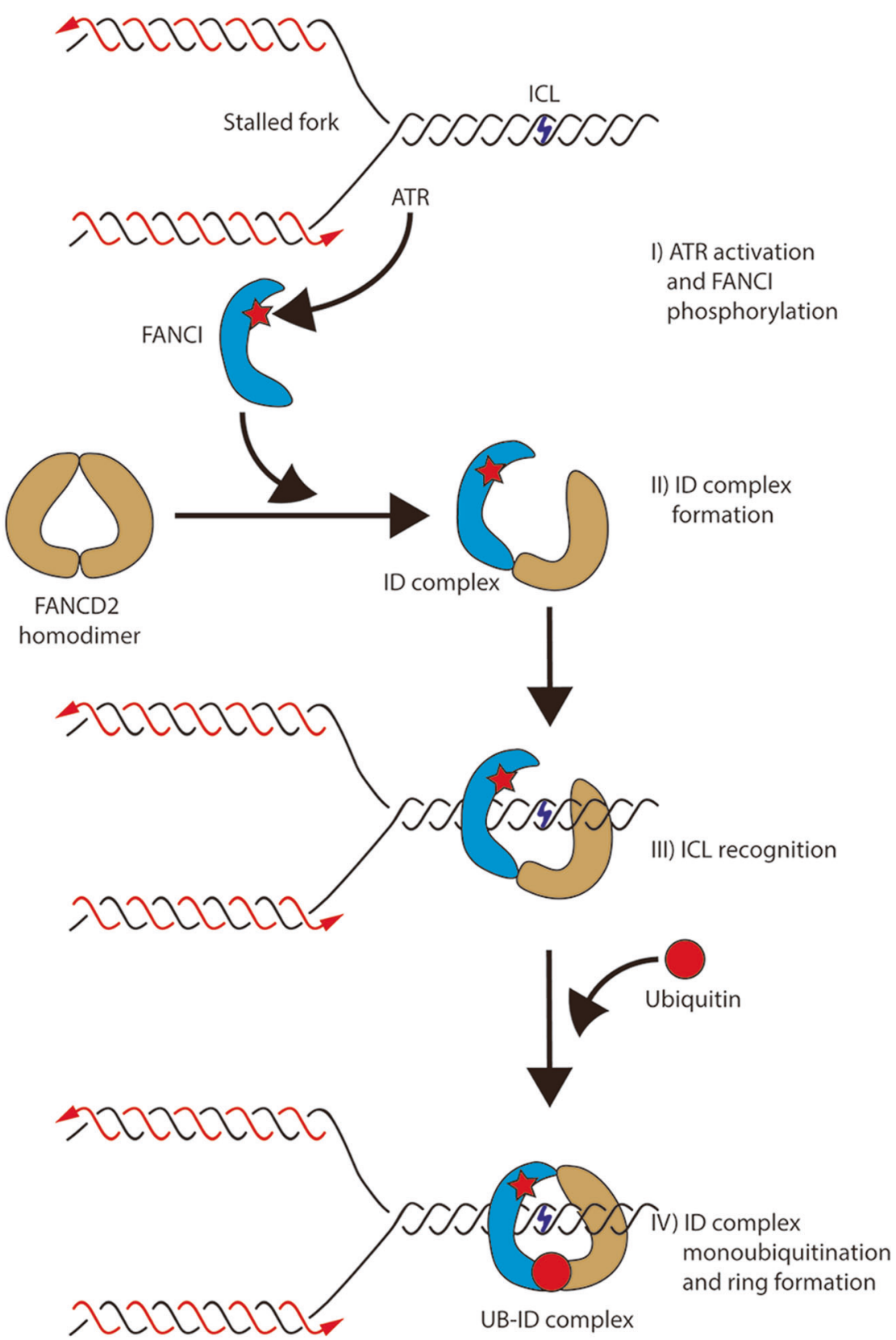

Fig. 1 ID complex forms circular clamp at the ICL upon monoubiquitination. Stalled replication fork activates the FA pathway through ATRmediated phosphorylation of $\mathrm{FANCl}$. Phosphorylated $\mathrm{FANCl}$, disrupts FANCD2 homodimer to form a heterodimer complex, which subsequently binds to ICL. FA core unit mono-ubiquitinates FANCD2, triggering conformational changes in the ID complex from an open channel to closed clamp.

implications. Once ubiquitinated, the ID complex loses its specificity for damaged bases or for branched DNA structures, a crucial step for damage recognition in the first place. The ub-ID clamp can then slide on DNA, perhaps contributing to removal of DNA-bound proteins to facilitate DNA repair and allow RAD51 filament formation. The establishment of ub-ID, long nucleoprotein filaments, might also contribute to protection of stalled replication forks, suppressing aberrant fork transitions. Analogously, halted transcription complexes at ICL can lead to the formation of R-loops due to RNA pol II backtracking, and the ID complex might hinder R-loop extension by binding to the RNA tails. The crosstalk between the ATR-dependent phosphorylation of $\mathrm{FANCl}$ and the ubiquitin-mediated regulation of the ID clamp represents a new paradigm in the field of DNA repair.

\section{REFERENCES}

1. Yuan, F., El Hokayem, J., Zhou, W. \& Zhang, Y. J. Biol. Chem. 284, 24443-24452 (2009).

2. Wang, R., Wang, S., Dhar, A., Peralta, C. \& Pavletich, N. P. Nature 580, 278-282 (2020).

3. Alcon, P. et al. Nat. Struct. Mol. Biol. 27, 240-248 (2020).

4. Joo, W. et al. Science 333, 312-316 (2011).

5. Lopez-Martinez, D. et al. Cell Rep. 27, 2990-3005 (2019).

6. Ishiai, M. et al. Nat. Struct. Mol. Biol. 15, 1138-1146 (2008).

7. Tan, W. et al. Elife 9, e54128 (2020).

8. Kim, J. M. et al. Dev. Cell 16, 314-320 (2009).

9. Liang, F. et al. Nat. Commun. 10, 2849 (2019).

10. Tan, W., van Twest, S., Murphy, V. J. \& Deans, A. J. Front. Cell Dev. Biol. 8, 2 (2020).

11. Zadorozhny, K. et al. Cell Rep. 21, 333-340 (2017).

12. Liang, Z. et al. Cell Rep. 26, 564-572 (2019). 